\title{
The Challenge of Tonal Fan Noise Prediction for an Aircraft Engine in Flight
}

\author{
Carolin Kissner $^{1)}$, Sébastien Guérin ${ }^{1)}$, Lars Enghardt ${ }^{1)}$, Henri Siller ${ }^{1)}$, Michael Pott-Pollenske ${ }^{2)}$ \\ 1) German Aerospace Center (DLR), Institute of Propulsion Technology, \\ Engine Acoustics, Müller-Breslau-Str. 8, 10623 Berlin, Germany. \\ 2) German Aerospace Center (DLR), Institute of Aerodynamics and Flow Technology, \\ Technical Acoustics, Lilienthalpl. 7, 38108 Braunschweig, Germany.
}

\section{Summary}

Expensive fly-over tests are needed to verify that noise certification standards are fulfilled. Currently, no numerical alternative exists to perform a holistic "virtual fly-over" test. As a step towards enabling such evaluations in the future, the authors focus on an isolated noise source - the tonal rotor-stator-interaction (RSI) of the fan stage. A high-fidelity simulation relying on a state-of-the-art yet computationally efficient method is performed for a V2527 aircraft engine in approach conditions. The computational domain includes the noise generation in the fan stage, its propagation in the engine inlet and bypass duct, as well as its radiation into the far field. Installation effects due to bifurcations and struts in the duct, ESS (engine section stator), liners, and inflow distortions are not considered. Post-processing methods are introduced and applied to the numerical data to allow for a meaningful comparison of the results to microphone data recorded during fly-over experiments. In particular, great care is taken to quantify the numerical dissipation of the simulation inside the nacelle and to enable a suitable correction of the numerical data. The numerical simulation cannot fully reproduce the experimental data indicating that its level of complexity is not yet sufficient. As there is no obvious cause for the mismatch, it would be necessary to incrementally increase the complexity of the simulation in order to pinpoint the most significant sources and effects.

\section{Introduction}

To ensure compliance with current noise regulations, new aircraft have to be acoustically certified with flyover noise tests according to procedures defined in the Annex 16 to the Convention on International Civil Aviation - Environmental Protection, Vol. I - Aircraft Noise [1]. Noise standards have to be met at the operating points of approach, sideline, and cutback. In the future, noise certification regulations will become even more stringent. A high-fidelity, nu- merical alternative to expensive fly-over noise tests, which are typically performed in the last stages of development just before an aircraft enters into commercial service, could enhance the aircraft design process. During "virtual fly-overs", relevant noise sources could be identified and quantified.

Some numerical studies that investigate the airframe noise of an aircraft in flight were recently conducted using a lattice Boltzmann flow simulation in combination with a Ffwocs Williams-Hawkings (FWH) noise propagation scheme $[2,3,4,5]$. All simulations were conducted for a Gulfstream G550 in landing configuration and neglect the contribution of the engine entirely. Good agreement in terms of surface pressures and far field noise between simulation data and windtunnel or fly-over measurements was demonstrated. However, fly-over noise tests required for certification are not limited to airframe noise. While the airframe noise is, in fact, more significant during landing than during take-off, the engine noise is thought to be slightly dominant or of a similar magnitude [6]. Thus, there is currently no comprehensive, numerical approach that can match the results of fly-over noise tests. In this paper, the authors chose a different approach for tackling the same problem. To allow for the use of "virtual fly-overs" in the design phase, such computations would need to be as accurate as possible while not demanding excessive amounts of computational resources. Thus, the complexity of such a computation would ideally be reduced to only include the noise sources and effects that have the most significant contribution at the certification points. As a first step towards pinpointing the most significant noise sources and determining the required complexity to achieve such a reduced yet accurate prediction, the authors decided to investigate one significant noise source, namely the rotor-stator-interaction tones of the fan, using a stateof-the-art, computationally efficient simulation technique. Tonal rotor-stator-interaction noise in the fan, which is produced by the interaction of rotor wakes with the downstream stator row, is a dominant engine noise source. The investigated aircraft configu- 
ration is the DLR ATRA research aircraft, an Airbus A320-232 equipped with two V2527-A5 engines. This paper strives to answer the question if a simulation relying on state-of-the-art yet relatively economical techniques can reproduce the fan tones observed during a fly-over of this aircraft at approach conditions.

The high-fidelity Computational Fluid Dynamics (CFD) simulation used the Harmonic Balance technique [7] and phase-shift, i.e. chorochronic, boundary conditions to simulate the tonal RSI noise for the fan of a full-scale V2527 engine. The computation considers the noise generation mechanism within the fan stage, its propagation through the engine's inlet and bypass duct, and its radiation to the far field. Lastly, the sound is radiated to observer positions on the ground using a FWH integral method [8]. The approach was successfully used in the past by Holewa et al.[9] to assess the fan tones of a configuration under test bed conditions. Transonic as well as subsonic operating points were assessed. For dominant modes, the simulation results differed by less than $3 \mathrm{~dB}$ from the measured data in the far field near the engine inlet and in the bypass duct. The experimental modal spectra contained generally weak, additional azimuthal modes, which were not caused by the rotor-stator-interaction. Only at the inlet at subsonic, approach-like conditions, the RSI mode was poorly radiated in the simulation and in the noise test. The experimental data was thus dominated by the additional modes and the sound pressure levels in the forward arc were underestimated in the simulation.

To allow for a meaningful comparison of the numerically determined tonal fan noise to the fan tones extracted from experimental data of a fly-over, the application of suitable post-processing techniques for both numerical and experimental data is essential. A particular focus of this paper will be the postprocessing of the numerical results. Correction procedures to account for numerical dissipation in the simulation were developed and applied. In addition, the numerical data was averaged over a range of directivity angles to emulate the post-processing of experimental data. Lastly, an equivalent monopole source was determined so that the numerical directivity resembles the directivity typically observed in fly-over experiments.

This paper will be structured as follows: At first, the fly-over measurements will be discussed including a description of the two different experimental setups and of the post-processing applied to the measured data. The setup of the numerical simulation and mesh generation procedure will be explained next. A focus of this paper will be the extraction and postprocessing of the numerical data, which will be discussed in a separate section. Lastly, the numerical data will be compared to the experimental data at the first three blade passing frequencies (BPF's) and the results will be discussed.
Table 1: Overview of flight conditions

\begin{tabular}{ccccc}
\hline & $\begin{array}{c}\text { rot. } \\
\text { speed } \\
N_{1}[\%]\end{array}$ & $\begin{array}{c}\text { flap/ } \\
\text { slat } \\
\text { angles }\end{array}$ & $\begin{array}{c}\text { land- } \\
\text { ing } \\
\text { gear }\end{array}$ & $\begin{array}{c}\text { true } \\
\text { air- } \\
\text { speed [kts] }\end{array}$ \\
\hline \hline Num. & 53.42 & - & - & 133 \\
\hline Exp. A & 50.9 & $27^{\circ} / 40^{\circ}$ & down & 139 \\
\hline Exp. B & 53.0 & $22^{\circ} / 20^{\circ}$ & up & $160-165$ \\
\hline \hline & & & & \\
& & & & \\
& & & & \\
& & & & \\
& & &
\end{tabular}

Figure 1: Sketch of experimental setups. Elliptical array denoted by letter A. Linear array denoted by letter B.

\section{Fly-over measurements}

Fly-over measurements were performed by two different groups of researchers, each using a different experimental setup. The two different experimental setups and their respective data will hereafter be denoted by experiment $A$ and experiment $B$. To get a more complete description, the authors chose the flights in each experimental data set, that most closely matched the numerical operating conditions in terms of rotational speed and true airspeed (see Table 1). The numerically imposed operating conditions during approach were determined using a performance cycle model defined by Wolters et al. [10]. It should, however, be noted that the deployment angles of the high lift devices and landing gear settings differ between experiments $\mathrm{A}$ and $\mathrm{B}$.

In the following subsections, the experimental setups of experiments A and B as well as the postprocessing of their respective data sets are briefly described.

\subsection{Description of experimental se- tups}

Two teams of researchers conducted fly-over measurements on DLR's Advanced Technology Research Aircraft (ATRA). Both teams, whose experiments are denoted by letters A and B, used different experimental setups.

For experiment A, an elliptical multi-arm array was 
positioned slightly starboard of the flight path in order to avoid the symmetry plane of the aircraft, as can be seen in Figure 1. The array contained 248 electret microphones and covered an area of $43 \mathrm{~m}$ by $36 \mathrm{~m}$. The longer axis was oriented parallel to the direction of flight in order to increase the resolution to enable the use of beamforming for source localization. Only one fly-over was recorded at the specified operating conditions. More details regarding the experimental setup can be found in Siller et al. [11, 12].

For experiment B, a linear microphone array was positioned perpendicular to the flight path (see Figure 1). The array consisted of nine microphones mounted on the ground. A similar setup was used by PottPollenske et al. [13] for studying a different aircraft configuration. Only the three center microphones of the array, that were positioned either directly or nearly underneath the flight path, were considered for the analyses presented here. Due to the greater distance from the outer microphones to the noise source, the narrow band spectra are dominated by the correction for the atmospheric absorption at frequencies of interest for the extraction of fan tones, i.e. at frequencies close to or lower than the third harmonic of the blade passing frequency. The comparatively low number of microphones that could be used for averaging the spectra was partly offset by the fact that data for nine, almost identical flights were provided. Thus, spectra at the different positions were averaged over the number of flights before averaging the spectra over the number of microphones. Therefore, a higher statistical precision could be reached.

\subsection{Post-processing of experimental data}

The microphone signals were corrected in the time domain to remove the frequency shift due to the Doppler effect. Then, the tones and respective background noise levels were extracted at the harmonics of the blade passing frequency.

All narrow band spectra were adjusted to remove the atmospheric absorption according to ISO standard 9613-1 [14], which is based on the modeling of molecular phenomena to derive analytical expressions for the atmospheric attenuation as introduced by Bass et al. [15]. An overcompensation of the sound pressure levels at high frequencies occurs when the spectral levels meet the noise floor. Thus, the measured levels remain approximately constant, while the corrected levels increase with an increasing frequency.

Further corrections were introduced to enable a comparison with the numerical data. All experimental spectra were corrected to account for the discrepancy in rotational speed $\Delta_{N}$ compared to the numerical settings using a scaling law derived from Heid- mann and Feiler's work [16]:

$$
\Delta_{N}=50 \log _{10}\left(\frac{N_{\text {num }}}{N_{\text {exp }}}\right) .
$$

Here, $N_{\text {num }}$ is the rotational speed of the simulation, while $N_{\text {exp }}$ denotes the rotational speed of the experiments. For experiment $\mathrm{A}, \Delta_{N}$ is equal to $1.05 \mathrm{~dB}$. For experiment B, $\Delta_{N}$ is equal to $0.17 \mathrm{~dB}$. It should be noted that Equation 1 agrees with a non-compact dipole-like source characteristic, which is reasonable for the rotor-stator-interaction tones of a fan but not necessarily for other broadband noise sources of an aircraft in flight. In addition, numerical and experimental far field data had to be normalized to the same observer distance. An observer radius of $100 \mathrm{~m}$ was chosen for the analysis of the numerical data. A correction $\Delta_{R}$ for the experimental data can be determined by

$$
\Delta_{R}=20 \log _{10}\left(\frac{R_{n u m}}{R_{\text {exp }}}\right),
$$

where $R_{n u m}$ denotes the numerical observer distance and $R_{\text {exp }}$, the experimental observer distance.

In the end, the authors aim to compare the sound pressure levels as a function of the directivity angles for the first three harmonics of the BPF. The directivity angles are defined so that $\theta=0^{\circ}$ corresponds to the observer position in the forward arc upstream of the aircraft. Conversely, a directivity angle of $\theta=180^{\circ}$ corresponds to the observer position in the rear arc downstream of the aircraft. The tones along with the respective background noise levels were extracted from the given narrow band spectra. Since the fan tones are scattered over several frequencies, the absolute sound pressure levels were determined by a summation over the respective bands and several of their neighboring bands. The background noise level was extracted at the bottom of the tonal peaks. The results are shown in Figure 2. It should be noted that some tones could not be identified in the spectra, particularly at higher frequencies and at increasing directivity angles. The dominant tone occurs at the 1st harmonic of the BPF. The tones at the higher harmonics are only slightly lower in amplitude. The amplitudes of the sound pressure levels of tones and background noise do not vary much with respect to the directivity angle. Therefore, a tendency towards a monopole-like directivity can be observed.

The differences in data of experiments $\mathrm{A}$ and $\mathrm{B}$ shall not be discussed in detail as such a discussion is outside the scope of this paper. It is expected that most of the observed differences are due to different settings of the high-lift devices, different pitch angles, and due to the landing gear (see Table 1). It should also be noted that there are some uncertainties regarding the experimental data. For some microphones, wooden tiles were placed under the microphones, which could 


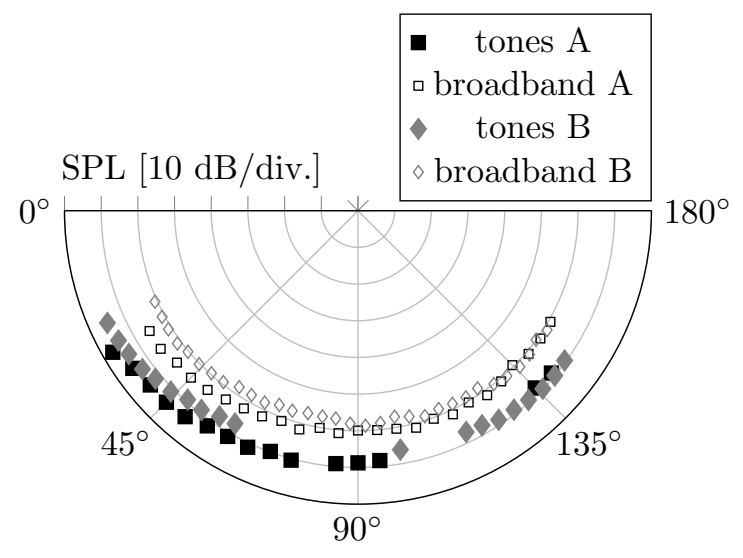

(a)

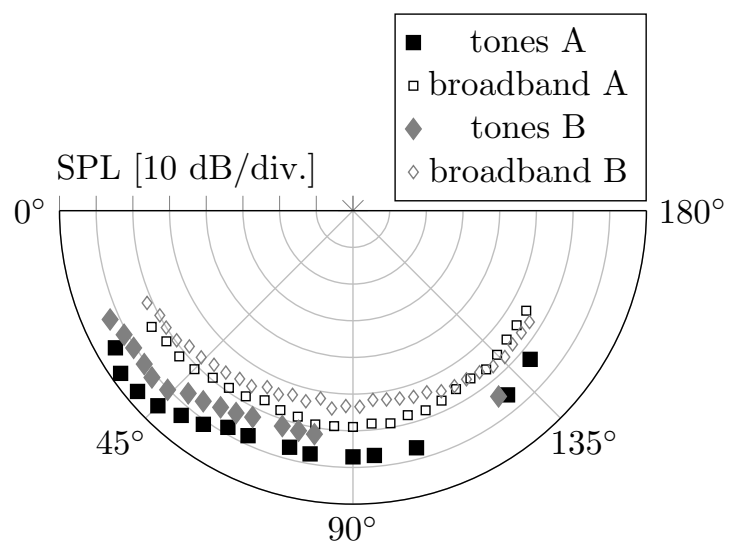

(b)

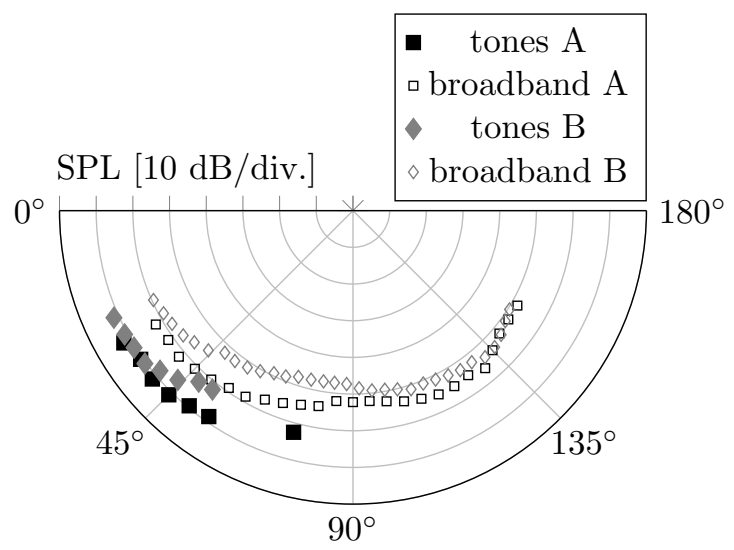

(c)

Figure 2: Experimentally determined directivity of tonal and background noise at a) BPF 1, b) BPF 2, and c) BPF 3. lead to refraction effects. The error should be within 1 to $2 \mathrm{~dB}$. In addition, some difficulties were encountered at this flight condition while determining the flight path using a combination of laser distance meters and Global Navigation Satellite System (GNSS) data from the aircraft. This could lead to small errors in de-Dopplerizing the spectra. Nonetheless, errors are expected to be smaller than $1 \mathrm{~dB}$.

\section{Numerical simulation}

In the following section, the numerical setup will be introduced and the used mesh generation procedure will be outlined.

\section{$3.1 \quad$ Setup}

The in-house CFD solver for internal flows, TRACE [17], was used to simulate the tonal fan noise of the V2527 engine at approach. The turbulence was modeled with the Menter SST $k-\omega$ model [18] and the spatial discretization was done via a Monotonic Upstream Scheme for Conservation Laws (MUSCL) approach [19] of second order accuracy based on Fromm's scheme. The main emphasis in setting up this simulation was to accurately predict the tonal fan noise as efficiently as possible. Therefore, the Harmonic Balance (HB) technique [7] and a phase-shift boundary condition [20] were applied.

The HB technique is a non-linear approach, which solves the unsteady Reynolds Averaged Navier-Stokes (uRANS) equations in the frequency domain rather than in the time domain. The main advantage is that this tends to be more efficient, especially when solutions are only needed at blade passing frequencies as is the case in the paper. In fact, both Frey et al. [21] and Holewa et al. [22] have shown that a HB simulation runs faster than a traditional uRANS simulation. Frey et al. [21] simulated the tonal fan noise of DLR's Ultra High Bypass Ratio (UHBR) at the second blade passing frequency in approach conditions. In addition to its computational efficiency, the authors were able to prove that the HB results match well with uRANS and experimental results. Holewa et al. [22] used the approach to study the impact of the interaction of steady hot streaks with a high-pressure turbine stage on the overall tonal noise. They were able to show that the HB simulation, which was designed to resolve harmonics 1 and 2 of the BPF, ran 20 times faster than the uRANS simulation. It was additionally observed that the phase-shift boundary condition and the non-reflecting in- and outflow boundary conditions are more robust in combination with the HB technique leading to an accelerated convergence.

The phase-shift boundary condition exploits the rotational symmetry of the blade rows in turbomachinery. For this simulation, the fan stage was reduced to single-passage rotor, outlet guide vane (OGV), and 


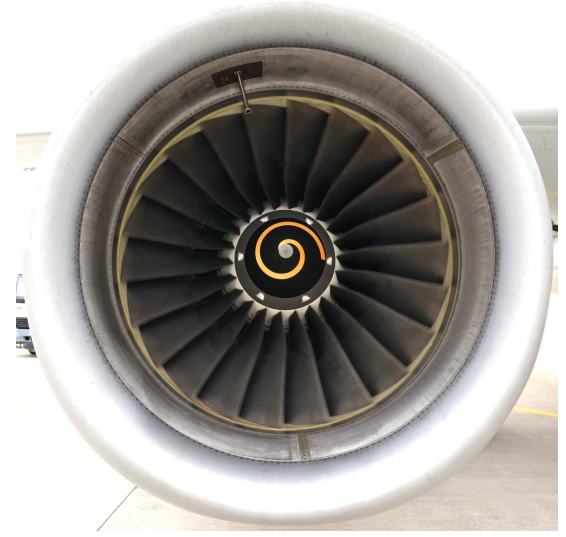

(a)

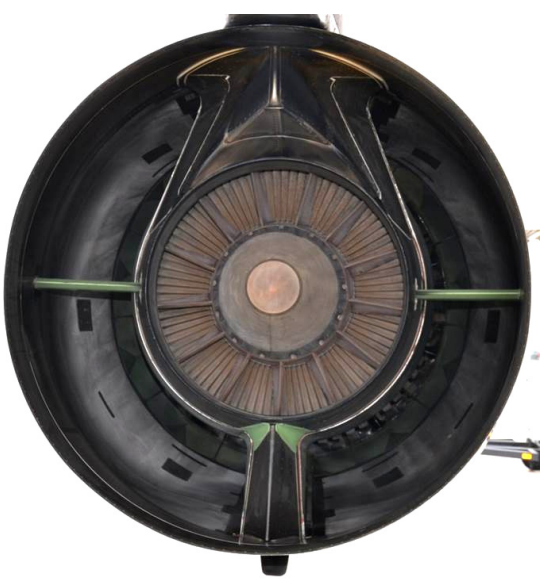

(b)

Figure 3: Complexity of engine configuration, which includes a) a spliced liner and a P2T2 sensor in the engine inlet and b) struts and bifurcations in the bypass duct (photos courtesy of Lech Modrzejewski, 2013).

engine section stator (ESS) blocks. While included in the simulation, the ESS was only considered for computing the mean flow. The implication of neglecting the ESS as a potentially relevant acoustic source in the simulation is included in the discussion section as one possible explanation for the discrepancy between the experimental and numerical data.

The phase-shift boundary condition was also applied in the engine inlet, bypass duct, and far field allowing for a reduction of the computational domain to two cells in the circumferential direction. The complex engine configuration, which is illustrated in Figure 3 was simplified to facilitate such a reduced, rotationally symmetric domain. The drooped inlet, bifurcations and struts in the bypass duct, the P2T2 probe and spliced liner in the inlet duct were not accounted for. As the inflow distortion due to the incidence angle of the nacelle decreases almost exponentially in the inlet section of an engine at moderate angle of attack [23], it was assumed that its impact as a new source of sound can be neglected. This assumption as well as other relevant assumptions are reconsidered in the discussion section in light of this paper's results and in the context of other recent studies. To prevent any flow detachment at the positions of the installations in the duct, the bypass geometry was slightly modified to keep the area of the duct constant in that section. Any acoustic liners and the engine core were also not included in the simulation.

\subsection{Mesh generation}

The mesh of a numerical simulation is always a compromise between accuracy and cost. A fine mesh reduces numerical dispersion and dissipation effects but is costly in terms of computational resources. A coarser mesh will be cheaper but result in a loss in accuracy. For this simulation, the authors pursued the following strategy: A best practice procedure, which was previously described by Kissner et al. [24], was applied with the aim of resolving the RSI tones with at least 40 points per wavelength (PPW) for 2 nd harmonic of the BPF. This resulted in a resolution of at least 30 PPW for 3rd of the BPF. Instead of doing a mesh study, numerical dissipation was quantified and used to correct the resulting far field noise data accordingly (see Subsection 4.2).

The current best practice for generating structured meshes is loosely based on the findings of Schnell [20], who performed an in-depth study of dissipation and dispersion effects for a two-dimensional test case. He determined that a mesh resolution of 20 to 25 points per wavelength $(\mathrm{PPW})$ resulted in a dissipation rate of $0.5 \mathrm{~dB}$ per wavelength perpendicular to the wavefront. However, a two-dimensional test case is not directly comparable to a fan stage. The Mach number and mesh resolution are not constant. In addition, a measure based on the wavefront is not practical as a wavefront changes depending on the mode and its position in the duct. To account for these uncertainties, an established practice is to aim for $40 \mathrm{PPW}$ in all directions for the acoustically cut-on modes. This is conservative compared to other meshing routines used by different authors $[25,26,27]$ in the community, who typically aim for a resolution of at least 20 to 25 PPW. Thus, the configuration studied in this work is comparatively rather well resolved. Nonetheless, numerical dissipation still occurs and accumulates due to the sheer size of the acoustically resolved domain, which not only includes the fan stage itself but also the entire bypass duct and portions of the far field. It is thus useful to analyze and quantify the dissipation in order to correct numerical results (see Subsection $4.2)$.

To design a suitable mesh for studying tonal fan noise, it is essential to know which acoustic modes are relevant. Acoustic, azimuthal modes resulting from RSI must fulfill the Tyler-Sofrin rule [28]

$$
m=h B+k V
$$


Table 2: Cut-on RSI modes

\begin{tabular}{ccc}
\hline $\begin{array}{c}\text { harmonic } \mathrm{h} / \\
\text { frequency f }[\mathrm{Hz}]\end{array}$ & $\begin{array}{c}\text { azimuthal } \\
\text { mode order } \mathrm{m}\end{array}$ & $\begin{array}{c}\text { radial mode } \\
\text { order } \mathrm{n}\end{array}$ \\
\hline \hline $1 / 1107$ & - & - \\
\hline $2 / 2213$ & -16 & $0-3$ \\
\hline $3 / 3320$ & 6 & $0-3$ (bypass) \\
& & $0-13$ (inlet) \\
\hline \hline
\end{tabular}

where $m$ describes the azimuthal mode order and $k$ represents an integer value. The harmonic of the $\mathrm{BPF}$, which can be determined by multiplying the rotational speed of the rotor $\Omega$ with the number of rotor fan blades $B(B P F=B \cdot \Omega)$, is denoted by $h$. For this configuration, the number of rotor fan blades $B$ is 22 and the number of stator fan blades $V$ is 60 . In the next step, it has to be analyzed, which of the determined Tyler-Sofrin modes are cut-on in the duct. The results are listed in Table 2. No modes are cut-on at BPF 1, while one azimuthal mode is cut-on at BPF's 2 and 3. A positive azimuthal mode order means that the mode rotates in the same direction as the rotor and vice versa. More radial modes are relevant in the inlet than in the bypass duct, since the duct area is larger in the inlet.

In order to achieve the desired resolution of $40 \mathrm{PPW}$ in all directions at harmonic 2 , the following approach was used: The wavelength in the axial direction was analytically determined at a position up- and downstream of the fan stage respectively in order to find the maximum cell size in axial direction. The mesh resolution in azimuthal direction was - in this case - dictated by aerodynamic rather than acoustic considerations, since resolving boundary layers and viscous wakes yields in more circumferential cells than would be required to resolve the relatively small azimuthal mode orders. In the radial direction, modes of a low radial order tend to be most energetic close to the tip radius. Thus, a clustering of the radial grid points near the tip radius is desirable. The radial grid resolution was chosen to be similar to comparable simulations and to avoid high cell aspect ratios, i.e. cells should have similar side lengths in all directions. The cells in the far field were designed to have approximately the same size as the cells within the duct in acoustically resolved regions. In regions near the boundaries of the far field, a cell stretching was introduced to suppress reflections at the far field boundary condition. The entire far field domain measures about 16.6 fan diameters in the axial direction and about 2.5 fan diameters in the radial direction. The acoustically resolved far field region begins more than two fan diameters upstream of the engine inlet and ends over five fan diameters downstream of the engine outlet. The resulting mesh contains about 25.9 million cells. The largest blocks are the rotor and
Table 3: Number of cells of used, chorochronic mesh and of an equivalent, full annulus mesh

\begin{tabular}{ccc}
\hline & chorochronic mesh & full annulus mesh \\
\hline \hline rotor & $12.4 \mathrm{M}$ & $272.7 \mathrm{M}$ \\
\hline OGV & $2.9 \mathrm{M}$ & $172.0 \mathrm{M}$ \\
\hline ESS & $0.4 \mathrm{M}$ & $42.2 \mathrm{M}$ \\
\hline far field & $10.2 \mathrm{M}$ & $7284.6 \mathrm{M}$ \\
\hline \hline total & $25.9 \mathrm{M}$ & $7771.6 \mathrm{M}$ \\
\hline \hline
\end{tabular}

far field blocks, each containing more than 10 million cells. Without the chorochronic boundary condition, a mesh of an equivalent resolution would contain over 7.7 billion cells (see Table 3), featuring 500 millions in the fan stage alone. On the one hand, this illustrates the fine mesh resolution of this setup. On the other hand, a simulation using an equivalent mesh spanning the full annular duct would have been impossible to perform on the current computing infrastructure. The used mesh resolution and the size of the computational domain would have had to be significantly reduced both in the zone of the fan stage and in the far field. The simplification of the configuration to rotationally symmetric computational domain is thus a priori a sensible choice to drastically reduce the computational time.

\section{Post-processing of numerical data}

To allow for a comparison of the numerically determined and measured fan tones, the numerical data had to be extracted in the far field and to be corrected to account for numerical dissipation effects and the total number of engines. In addition, the numerical data was post-processed to emulate the postprocessing and the characteristic, monopole-like directivity of the experimental data.

\subsection{Determination of far field directiv- ity}

To investigate far field noise, the FWH method based on the work of Weckmüller et al. [8] was applied. In order to receive reasonable results, the choice and positioning of the FWH integration surfaces, where the pressure perturbations are extracted and propagated to the observer positions, is essential. The FWH approach assumes constant mean flow at the position of the integration surfaces and in order to capture all of the emitted sound, the surface should enclose the sound source. These aims are easily met near the inlet of the engine but prove to be a challenge near the outlet of the engine. A closed integration surface would cut through the shear layer between ambient air 


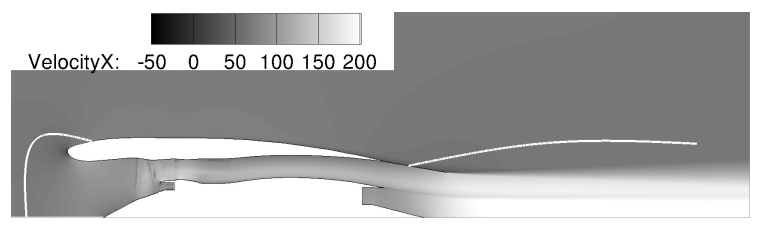

(a)

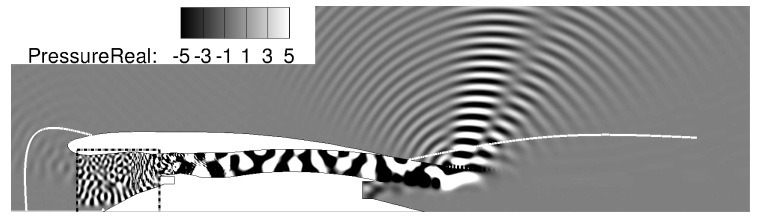

(b)

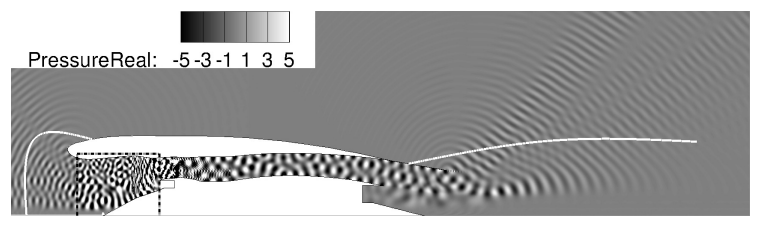

(c)

Figure 4: FWH integration surfaces shown for a) mean flow, b) BPF2, c) and BPF3. The box signifies the rotating block, which contains all harmonics of the BPF.

and bypass flow and through the shear layer between the bypass and core flows. An open integration does not fully enclose the sound source and emitted sound could potentially be neglected. The authors chose the latter option in order to not violate the constant flow assumption of the FWH technique. In addition, a comparison of the two variants showed that the deviation in the overall sound power levels was less than $0.25 \mathrm{~dB}$ at BPF 2 and BPF 3 (see Kissner et al. [24]) with little differences in the directivity of the sound pressure. The FWH integration surfaces are shown in Figure 4. The 180 observer positions were placed in a semicircle of radius $100 \mathrm{~m}$ around the engine.

The FWH method outputs real $p_{\text {re }}$ and imaginary fluctuating pressures $p_{i m}$ at each sensor position, which can be used to determine sound pressure levels:

$$
S P L[d B]=10 \cdot \log _{10}\left(\frac{p_{r m s}^{2}}{p_{r e f}^{2}}\right) .
$$

The reference pressure $p_{r m s}$ is equal to $2 \cdot 10^{-5} \mathrm{~Pa}$. The root mean square pressure $p_{r m s}$ is determined by

$$
p_{\text {rms }}=\frac{\sqrt{2 \cdot\left(\left(p_{\text {re }, \text { in }}+p_{\text {re }, \text { out }}\right)^{2}\right.}}{\left.+\left(p_{\text {im }, \text { in }}+p_{\text {im }, \text { out }}\right)^{2}\right)},
$$

where $p_{r e}$ and $p_{i m}$ are the real and imaginary part of the Fourier coefficients of the complex pressure field for a two-sided spectrum, the subscript in denotes the contribution from the engine inlet, and the subscript out, the contribution from the engine outlet. The resulting sound pressure levels are shown as

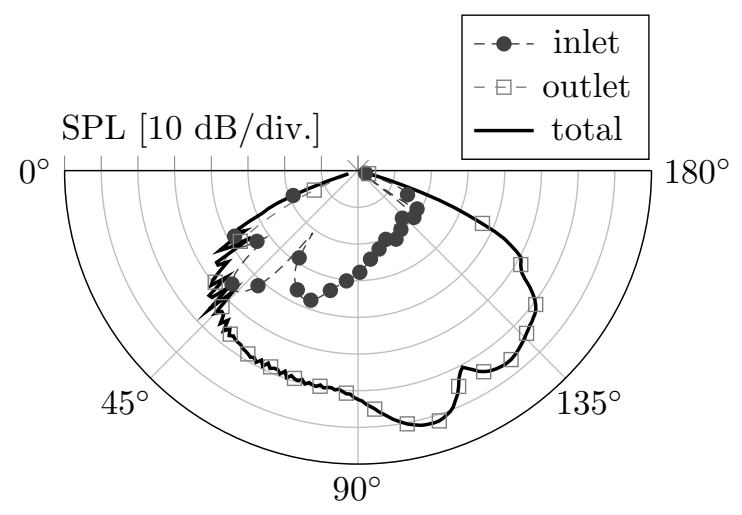

(a)

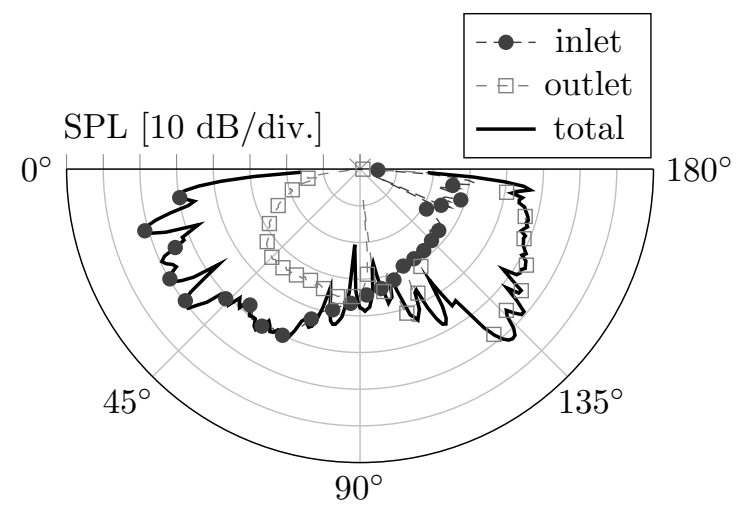

(b)

Figure 5: Numerically determined directivity of fan tones at a) BPF 2 and b) BPF 3 .

a function of the directivity angle $\theta$ at harmonics 2 and 3 of the BPF in Figure 5 . It can be seen that the overall noise emitted at BPF 2 is dominated by the emission at the engine outlet. At directivity angles near $40^{\circ}$, the total sound pressure line becomes jagged. This can occur, if the sound pressure originating from engine inlet and outlet are of a similar magnitude but have a different phase. At BPF 3, contributions from both inlet and outlet influence the overall directivity.

\subsection{Consideration of numerical dissi- pation}

To analyze the numerical dissipation, the sound power was first determined at different positions in the duct to identify trends and observe insightful phenomena. In the second step, a correction was formulated and applied to the far field data.

To determine the sound power within the duct, the extended plane pressure mode matching technique of Wohlbrandt et al. [29] was used. The technique can distinguish between sound and unsteady fluctuating pressures with convective characteristics. It relies on the assumption of uniform flow and of slowly changing duct contours. For the analysis with swirl, which 


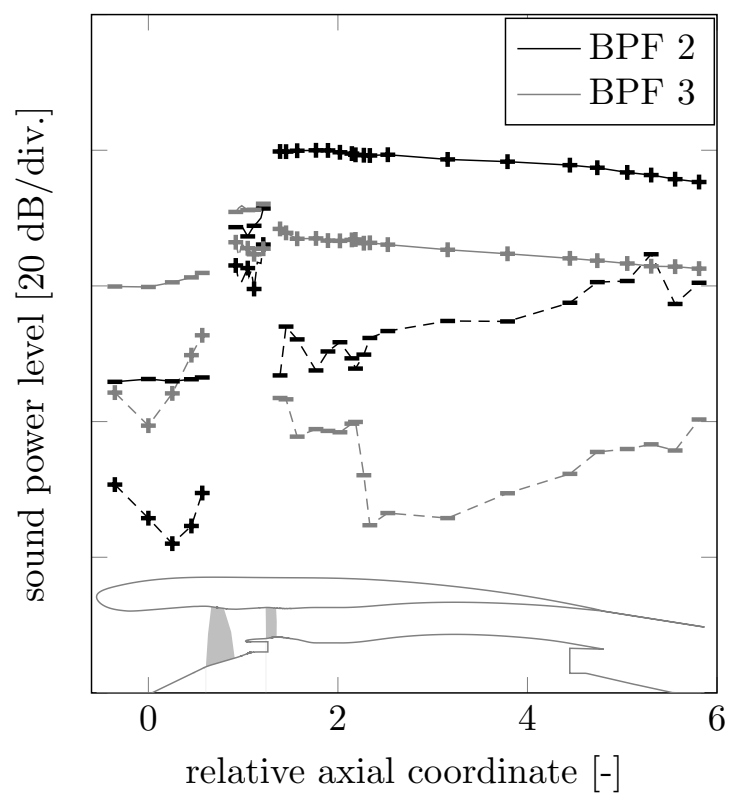

Figure 6: Sound power levels in duct. Solid lines mark the sound waves emitted by the fan stage towards the duct ends. Reflections back to the fan stage are shown by dashed lines. The line markers indicate the propagation direction: "+"-markers denote a propagation in positive, axial direction, i.e. downstream. "-"-markers denote a propagation in negative, axial direction, i.e. upstream.

is present in the interstage, a solution for solid body swirl flows is applied. This mainly includes two extensions: i) the description of the acoustic modal solution in rotating mean flows following Morfey [30], ii) the calculation of an equivalent solid body swirl flow with the same net force, mass flow, angular momentum and enthalpy flow as the original CFD mean flow solution (method not published yet). Its acoustic model cannot consider radial flow and the aerodynamic model neglects the viscous dissipation of the perturbations and effects due to the wake expansion.

The sound power levels in the duct are shown in Figure 6. In this figure, solid lines mark the propagated sound, while reflections are shown by dashed lines. The line markers indicate the propagation direction: "+"-markers denote a propagation in positive, axial direction, i.e. downstream. "-"-markers denote a propagation in negative, axial direction, i.e. upstream. Particularly in the domains up- and downstream of the fan stage, the sound power levels of reflections are 10 to $35 \mathrm{~dB}$ smaller than prevalent sound power levels. This is to be expected for modes that are well cut-on. Furthermore, the main sound power levels decrease nearly linearly with an increasing distance from the fan stage. Since the dissipation behavior is linear and nearly all dissipation in a duct can be attributed to numerical dissipation, it can be quantified in terms of dissipation rates, which are listed in
Table 4: Dissipation rates in duct

\begin{tabular}{ccc}
\hline direction & $\begin{array}{c}\text { harmonic } \mathrm{h} \\
\text { of BPF }\end{array}$ & $\begin{array}{c}\text { dissipation rate } \\
{[\mathrm{dB} \text { per OGV radius] }}\end{array}$ \\
\hline \hline \multirow{2}{*}{2} & upstream & 0.5 \\
& downstream & 1.0 \\
\hline \multirow{2}{*}{3} & upstream & 2.2 \\
& downstream & 1.2 \\
\hline \hline
\end{tabular}

Table 4. The dissipation rates are given as decrease in sound power per OGV radius as the usual definition in PPW is not practical due to the large number of modes. It can be observed that the dissipation rates are higher for BPF 3 than for BPF 2. This is reasonable as resolving higher frequencies requires a finer grid resolution.

The sound power levels in the interstage section of the duct are insightful as well. It proves that the sound originates at the stator vanes, since the sound power levels are higher in the upstream direction. Rotor shielding can also be clearly seen: as the sound originating from the OGV passes through the rotor row, its level drops off significantly. In addition, the decrease in sound power is about $10 \mathrm{~dB}$ larger for the BPF 2 than for the BPF 3. Two factors could contribute to such a shielding effect: Firstly, the swirl in the flow increases the tendency of an acoustic mode to become cut-off if the swirl Mach number and the circumferential mode have the same rotational direction as shown by Roger and Arbey for simplified solidbody swirl and free vortex flows [31]. Those observations were confirmed by e.g. Masson et al. [32] for realistic flows with swirl and shear. This described effect could impact the azimuthal mode order 6 at BPF 3. Secondly, azimuthal modes rotating in the same direction as the rotor are transmitted more effectively as demonstrated by Kaji and Okazaki [33]. This latter effect seems to be dominant here and explains why the shielding effect for mode -16 of BPF 2 is stronger than for mode 6 at BPF 3. The effects of the shielding effect can also be guessed from Figure 5, where the sound radiated from the inlet is less compared to the sound radiated from the engine outlet.

In the next step, a correction was formulated based on the previous findings regarding the numerical dissipation. The correction was applied to the fluctuating pressures used for computing the sound pressure levels:

$$
p_{\text {rms }}=\frac{\sqrt{2 \cdot\left(\left(f_{\text {in }} \cdot p_{\text {re }, \text { in }}+f_{\text {out }} \cdot p_{\text {re }, \text { out }}\right)^{2}\right.}}{\left.+\left(f_{\text {in }} \cdot p_{\text {im }, \text { in }}+f_{\text {out }} \cdot p_{\text {im }, \text { out }}\right)^{2}\right)}
$$

As can be seen in previous equation, correction fac- 
Table 5: Dissipation between fan stage and FWH surfaces in PWL

\begin{tabular}{ccc}
\hline dissipation $[\mathrm{dB}]$ & At inlet & At outlet \\
\hline \hline BPF $(\mathrm{h}=2)$ & $1.5 \mathrm{~dB}$ & $4.5 \mathrm{~dB}$ \\
\hline BPF $(\mathrm{h}=3)$ & $3.2 \mathrm{~dB}$ & $7.0 \mathrm{~dB}$ \\
\hline \hline
\end{tabular}

tors are applied. These are quantified as

$$
f_{i}=10^{\frac{\Delta_{\text {diss }, i}}{20}},
$$

where $\Delta_{\text {diss }, i}$ denotes the difference in sound power in decibels between the fan stage and the FWH integration surface. The $\Delta_{\text {diss }, i}$ - values for both BPF's at the in- and outlet of the engine are listed in Table 5. The correction equation and the definition of the correction factor make two inherent assumptions:

1. All dissipation is numerical dissipation.

2. The numerical dissipation has a linear behavior between the fan stage and the FWH integration surface.

Both assumptions are necessary simplifications of a more complex problem. Particularly near the outlet of the engines, not all dissipation will necessarily be numerical dissipation. Bechert [34] has shown that acoustic energy can be transformed into vortical energy in shear flows. This effect - if relevant for this case - will be neglected by the introduced correction. The impact of the correction of the sound pressure levels can be seen in Figure 7. The correction has a greater effect in the rear of the engine than at the inlet because the bypass duct is longer than the inlet duct and thus more overall numerical dissipation is induced.

\subsection{Correction for number of engines}

As the ATRA aircraft has two engines and the simulation only considered one engine, the sound pressure has to be corrected accordingly by the correct number of engines $n$ :

$$
S P L[d B]=10 \cdot \log _{10}\left(n \cdot \frac{p_{r m s, t o t a l}^{2}}{p_{r e f}^{2}}\right) .
$$

This correction assumes that both engines are uncorrelated sound sources. In Figure 7, it can be seen that the correction causes an increase in the sound pressure levels of $3 \mathrm{~dB}$.

\subsection{Smoothing of data}

The post-processing of fly-over measurements is more challenging than for static test beds or wind tunnels since the source is in motion. Particularly at

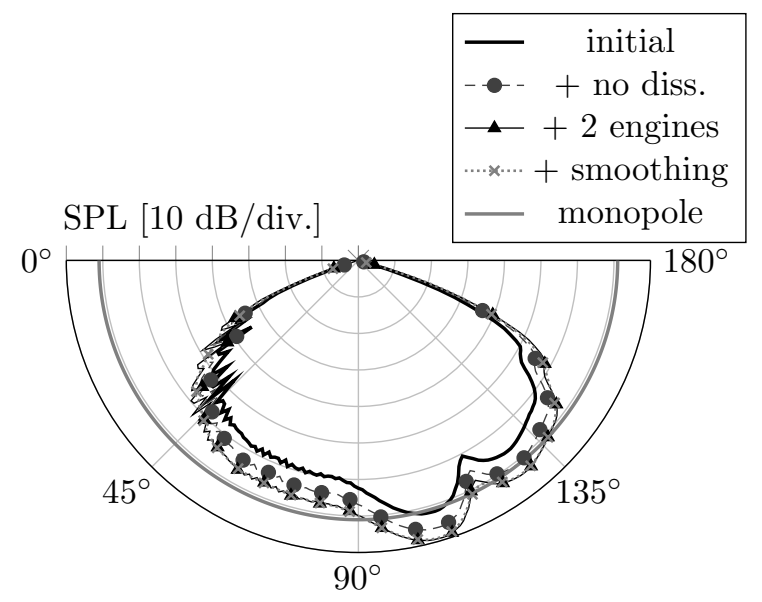

(a)

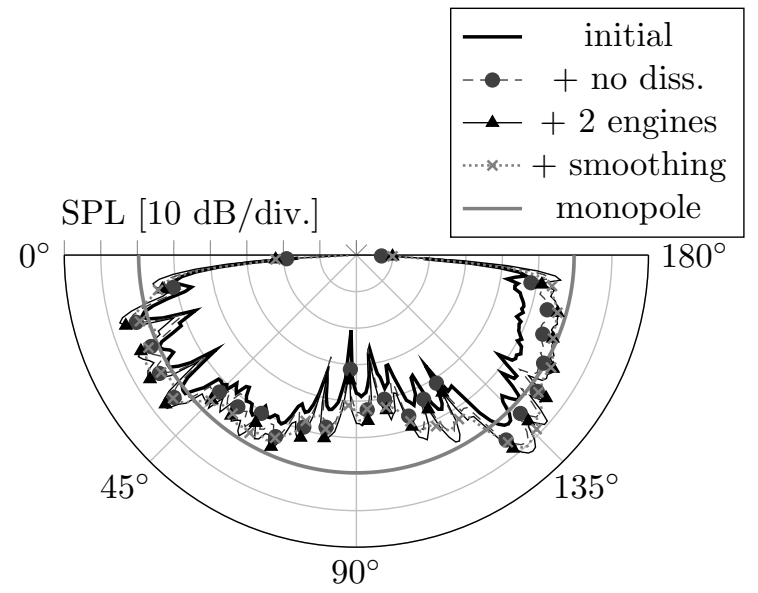

(b)

Figure 7: Numerically determined directivity of RSI tones and corrections at a) BPF 2 and b) BPF 3 .

a directivity angle of $90^{\circ}$, the available integration time reaches a minimum. To increase the statistical accuracy, an averaging over range of directivity angles is needed. Thus, there is built-in smoothing of the directivity information, which results from the post-processing of the experimental data. To reproduce this smoothing effect, the numerically determined sound pressure levels were smoothed by a convolution with a Gaussian bell function over a range of directivity angles. The chosen range of angles was three degrees in both directions, which approximately corresponds to the range of angles used in post-processing the experimental data. The effect can be seen in Figure 7.

\subsection{Determination of an equivalent monopole}

As previously shown in Figure 2, the experimental results display a monopole-like directivity behavior. In the numerical simulation, the directivity is pronounced and quite unlike a monopole. This obser- 
vation is quite reasonable. The simulation considers pure rotor-stator-interaction noise, which is characterized by one relevant cut-on mode at BPF's 2 and 3 . When isolated fan noise is considered experimentally in fan test rigs, the modes are generally dominated by the RSI modes only as shown by Heidelberg [35] and Holewa et al. [9]. Scattering mechanisms at installations within the duct like struts and bifurcations are neglected in the simulation. The interactions with installations would cause each mode to scatter into other modes. Whereas one single mode has a strong directivity, multiple modes - each having a strong but different directivity - result in a smoother directivity when added up. The more modes carry acoustic energy, the more the directivity becomes a monopole. It is assumed here that the overall sound power is unaffected by the scattering. Since the experimental data includes scattering effects, thus explaining its directivity characteristic, the numerical data does not. To emulate the experimental data, a monopole of equivalent sound power was determined for the numerical data. The sound power resulting from sound pressure $p$ was calculated by the equation

$$
P=\int_{S} I_{i} n_{i} d S
$$

where $n_{i}$ is the vector normal to the integration surface, $I_{i}$, the acoustic intensity vector, and dS, the elemental surface. An "equivalent" monopole has the same overall sound power. For small Mach numbers $M$, the following expression can be applied to calculate the root mean square pressure, which is constant at all directivity angles:

$$
p_{r m s} \simeq \sqrt{\frac{P \rho_{0} c_{0}}{4 \pi R^{2}}\left[\frac{1}{4 M} \ln \left(\frac{1+2 M}{1-2 M}\right)\right]^{-1}}, \forall M \ll 1
$$

Here, $\rho_{0}$ is the static pressure and $c_{0}$ denotes the speed of sound. $R$ describes the distance of the observers to the sound source. The equivalent monopoles are shown in Figure 7.

\section{Comparison of numerical and experimental far field data}

After applying corrections to the numerical data, a comparison of the numerically determined fan tones to the fan tones from the experiments is shown in Figure 8. It should be noted that all shown sound pressure levels are absolute levels for both numerical and experimental data. All directivity plots use the same dB-scale and can therefore be directly compared.

The difference between the numerical and experimental data is most striking at BPF 1 . In the experimental data, the fan tone at BPF 1 is cut-on and dominant. The simulation cannot reproduce this result as

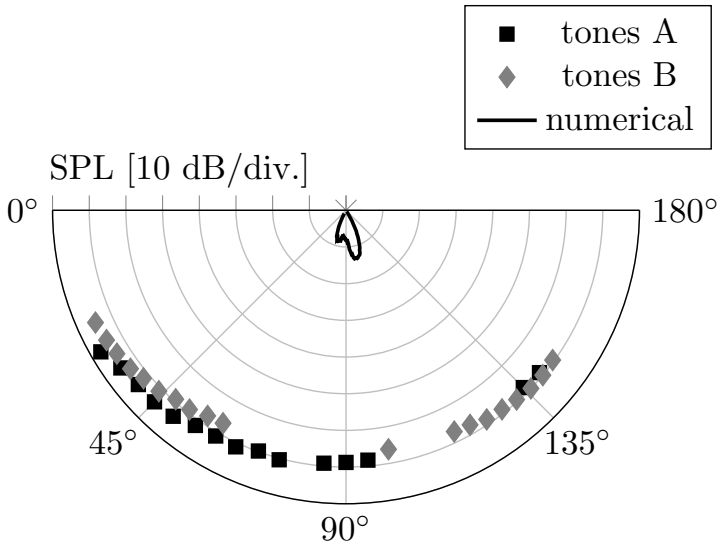

(a)

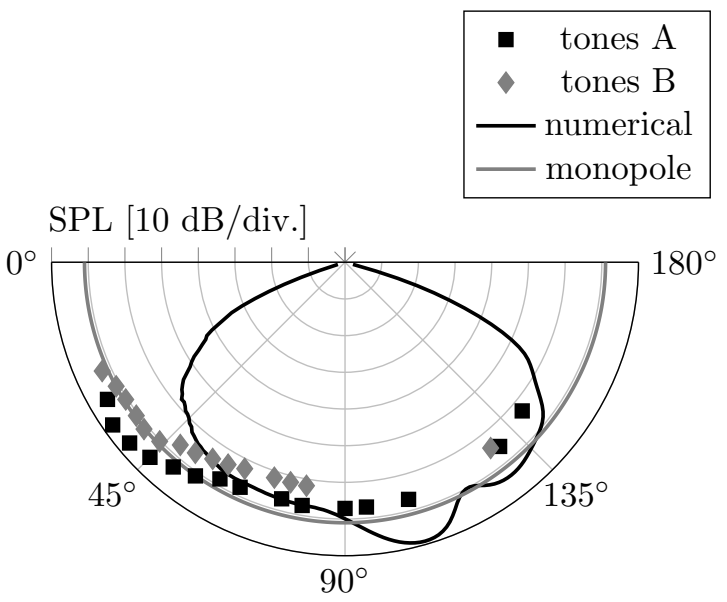

(b)

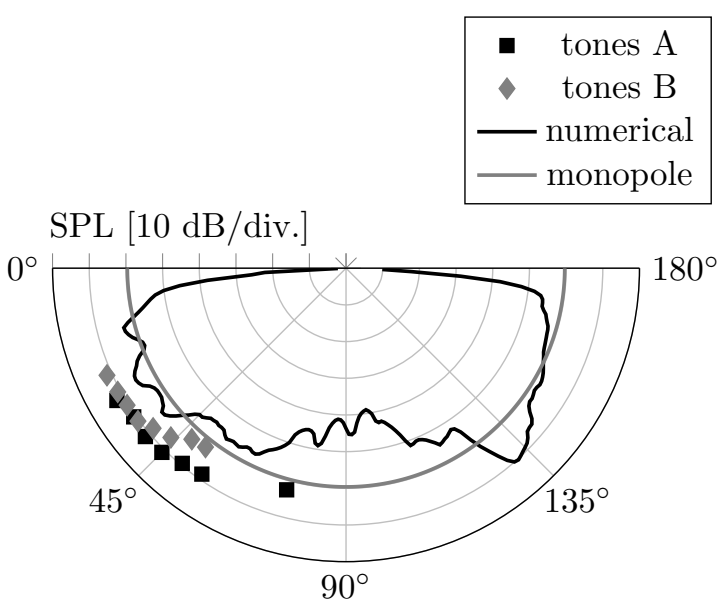

(c)

Figure 8: Comparison of numerically determined to measured RSI tones at a) BPF 1, b) BPF 2, and c) BPF 3. 
it only considers rotor-stator-interaction tones. In addition, the flow and the geometry are axisymmetric, thus eliminating the possibility of modal scattering, which would cause observed modes to differ from the Tyler and Sofrin's rule. As a consequence, there are no cut-on RSI modes at BPF 1 . The sound pressure detected in the simulation can be attributed to the low radiation effectivity of cut-off modes [36] and possibly to hydrodynamic perturbations originating in the wakes of the OGV and rotor blades. Thus, the strong mechanism that causes the fan tone at BPF 1 to be cut-on in the experiment is not included in the simulation.

As expected, the directivity patterns calculated numerically are characterized by the presence of pronounced lobes, which are typical if only few induct modes are contained in the radiated sound field. In contrast, the measured directivity is much smoother and, therefore, closer to a monopole-like behavior. While the amplitude of the sound pressure is comparable for the equivalent monopole and the experimental data at the 2nd harmonic of the BPF, this is not the case at 3rd harmonic of the BPF. In the simulation, there is also a significant difference in the overall magnitude of the numerically determined sound pressure between BPF's 2 and 3. This decrease is less pronounced in the measurements.

It can be concluded that the simulation is not yet adequate in terms of its complexity for accurately predicting the fan tones, in particular the directivity shapes, of a V2527 engine in flight.

Some effects that could potentially explain the discrepancies between the experimental and numerical fan tones include:

- Installation effects within the duct due to struts and bifurcations are not considered in the simulation. Research by Holewa et al. [37] and by Redonnet and Druon [38] suggest that bifurcations and struts cause a richer modal content of the RSI tones due to several mechanisms: i) sound generation by the rotor wake (vortical) interaction with those structural elements, ii) scattering of the acoustic waves into new modes as they propagate through obstacles, and iii) impact of the potential field of the bifurcations on the rotor wake structure before they interact with the stator. This can not only jeopardize a cut-off fan design as shown by Winkler et al. [39] but also result in a smoother directivity in the far field. In fact, the acoustic scattering at the bypass bifurcations suffices to significantly change the far field directivity as observed by Chen et al. [40]. They found that the directivity was less pronounced and the sound pressure levels increased by some decibels at some directivity angles compared to a simulation neglecting bifurcations. An increase in sound power due to an interaction of the rotor wakes with duct obstacles also depends on the separation distance.

- Only the fan tones resulting from the interaction of the rotor wakes with the fan OGV's was considered, whereas the contribution of the ESS was neglected. Results in the literature are not conclusive regarding the importance of the ESS contribution to the overall tonal fan noise, which lead to the hypothesis that the fan design and the applied operating conditions play a key role. Thus Holewa et al. [9] showed that the rotorESS-interaction tones were significantly smaller than the rotor-stator-interaction tones, despite the fact that the ESS is twice closer to the rotor than the OGVs. Winkler and al. [39] also made similar observations. Contradictorily, the results presented by Namgoong and Arisetti [41] show the presence of a strong acoustic field produced by the ESS at 2nd harmonic BPF, which propagates up into the bypass duct.

- Inflow distortions due to the incidence angle are not included in the simulation. The work of Guérin and Holewa [42] suggests that this effect is less important than others. Guérin and Holewa numerically investigated an inflow distortion due to a typical incidence angle at approach for a full annulus fan stage featuring a drooped inlet. They observed only a marginally higher acoustic power level compared to a simulation with a clean inflow. However, the SPL directivity was impacted by the distortion in the forward arc due to the asymmetric flow gradients at the inlet. Contrarily to that, the work done by Daroukh et al. [27] shows that the emitted sound of an engine considering an asymmetric intake can significantly increase - in their case by about $3 \mathrm{~dB}$ compared to an equivalent setup considering an axisymmetric intake. Also, the P2T2 sensor could create a tone by the interaction of its wake with the rotor.

- Liners are neglected in the computation. Even though liners exist to damp emitted acoustic waves, they can also have a negative impact on the noise emission if they are badly designed. Thus, the interaction of the rotating pressure field with liner splices in the duct inlet can cause energy to be transferred from non-propagating, rotor-locked pressure field modes into lower, cuton mode orders as discussed in the works of McAlpine and Wright [43] and Bassetti et al. [44]. Furthermore, liners are known to modify the directivity of the acoustic field radiated through the inlet [45] or the nozzle [46]. The degree of impact depends on the wave and liner characteristics.

- Another factor, which was not considered in the simulation, is that the engines of the ATRA research aircraft have been in service for many 
years. A degradation of the engine liners and changes of the engine geometry - particularly of the blade geometry - due to wear and maintenance work can alter the noise signature of an engine. In fact, acoustic methods for the detection of blade faults in turbomachinery [47, 48] analyze the altered sound emission by comparing it to a "healthy" configuration.

This list is not meant to be exhaustive but rather to emphasize the complexity of the problem at hand. Only future investigations can shed light onto important effects and sources, which need to be considered in order to achieve a better agreement.

\section{Conclusion}

Tonal rotor-stator-interaction (RSI) noise of the fan is thought to contribute significantly to the overall noise of a commercial aircraft. Therefore, the numerical calculation of this noise generation mechanism for an aircraft in flight is an important step towards a virtual acoustic certification. The objective of this paper was to investigate if a numerical simulation relying on state-of-the-art yet computationally efficient techniques can correctly predict the RSI tones observed during fly-over experiments of a conventional aircraft configuration.

The fly-overs were performed using the DLR A320 research aircraft, which is equipped with two V2527 engines. The assessed operating point was approach. The high-fidelity simulation considered the noise generation mechanism within the fan stage, the noise propagation within the engine's inlet and bypass duct, and the sound radiation into the far field. The airframe, liners within the engine, the engine core, and installations within the engine's inlet and bypass duct, i.e. bifurcations, struts, and installed sensors, were not included in the simulation. To keep the computational effort as reasonable as possible, the Harmonic Balance technique was applied. To enable the use of a phase-shift condition, the engine geometry had to be simplified to a rotationally symmetric domain. Thus, the drooped engine inlet and the incidence angle at approach were neglected.

In order to achieve a meaningful comparison of numerical to experimental data, the authors introduced post-processing techniques to correct the numerical results. A particular focus of this work was the consideration and quantification of numerical dissipation.

The corrections of the numerical data do, in fact, decrease the discrepancy between the experimental and numerical RSI tones. Nonetheless, the simulation fails to reproduce the fan tones - in terms of directivity and absolute sound pressure levels - of the fly-over measurements. While the simulation technique yields good results in well-controlled rig conditions [9], where fan noise is investigated as an isolated noise source, this is not the case for the fan noise of an aircraft in flight. Nevertheless, it can serve as a starting point for future investigations. The complexity of the simulation would need to be increased incrementally in order to pinpoint important influences on the fan noise. Ideally, a simulation would only be as complex as absolutely necessary to achieve suitable results.

Investigating the possible explanations between the numerical and experimental data as listed in section 5 would require an increase in the complexity of the simulation. The contribution of the ESS can be included without increasing the computational effort by a large amount as the simulation domain remains axisymmetric. Similarily, accounting for the presence of a spliceless liner - a now established technology for examining commercial aeroengines - is feasible using the same simplified geometry. In contrast, a full annulus simulation is required when including struts and bifurcations in the simulation. Such a full annulus simulation domain is significantly more expensive than a reduced, rotationally symmetric domain. Along with the ESS, struts and bifurcations are certainly the most promising candidates for generating a rich acoustic modal content to agree with the experimental observations: for the V2500 engine, this level of complexity seems necessary to capture the level of the BPF tone and the smooth directivity observed in the experimental data. The need to consider the drooped inlet and the nacelle incidence is also an open question, which becomes very relevant at high operating speed. Taking into account the effect of engine degradation on the acoustics is complicated as no study of this phenomenon exists for realistic fans. Mößner et al. [49] have recently studied the influence of mounting the V2500 engine underneath a fully equipped wing. The results show neither the shape of the directivity nor the magnitude of the sound pressure levels are significantly altered. Thus, the interaction of the fan tones with the wing - and likely also with the fuselage - cannot explain the observed discrepancies between numerical and experimental data.

Overall, the outcome of this investigation is rather sobering for the preliminary design of fan stages in general. It begs the question to what extent a fan stage can be acoustically designed and optimized by only considering an isolated, idealized fan stage and neglecting all other components. The simulation presented in this paper shows that the accurate consideration of tonal fan noise is not sufficient in capturing the noise of fly-over measurements. The further investigation of this issue is, therefore, not only essential for performing "virtual fly-overs" in the future but also for the evaluation and improvement of current design and optimization techniques. 


\section{References}

[1] ICAO, Committee on Aviation Environmental Protection. Annex 16 to the Convention on International Civil Aviation - Environmental Protection, Vol. I Aircraft Noise. Chicago Convention Annex, 2014.

[2] D. Casalino, S. Noelting, E. Fares, T. Van de Ven, F. Perot, and G. Bres. Towards numerical aircraft noise certification: Analysis of a full-scale landing gear in fly-over configuration. In 18th $A I A A / C E A S$ Aeroacoustics Conference, 2012.

[3] M. R. Khorrami, E. Fares, and D. Casalino. Towards full aircraft airframe noise prediction: lattice Boltzmann simulations. In 20th AIAA/CEAS Aeroacoustics Conference, 2014.

[4] E. Fares, D. Casalino, and M. R. Khorrami. Evaluation of Airframe Noise Reduction Concepts via Simulations Using a Lattice Boltzmann Approach. In 21st AIAA/CEAS Aeroacoustics Conference, 2015.

[5] E. Fares, B. Duda, and M. R. Khorrami. Airframe Noise Prediction of a Full Aircraft in Model and Full Scale Using a Lattice Boltzmann Approach. In 22nd AIAA/CEAS Aeroacoustics Conference, 2016.

[6] AIRBUS. Getting to Grips with Aircraft Noise. Technical report, Airbus Customer Services, 2003.

[7] C. Frey, G. Ashcroft, H.-P. Kersken, and C. Voigt. A harmonic balance technique for multistage turbomachinery applications. In ASME Turbo Expo 2014: Turbine Technical Conference and Exposition, 2014.

[8] C. Weckmüller, S. Guérin, J. Wellner, and U. Michel. Ffwocs Williams and Hawkings Formulation for the Convective Wave Equation and Permeable Data Surface. In 16th AIAA/CEAS Aeroacoustics Conference, 2010.

[9] A. Holewa, S. Guérin, L. Neuhaus, L. Danwang, and T. Huimin. Tones from an Aero-Engine Fan: Comparison between Harmonic-Balance Simulation and Experiment. In 22nd AIAA/CEAS Aeroacoustics Conference, 2016.

[10] F. Wolters, R. Becker, R. Schnell, and P.-B. Ebel. Engine performance simulation of the integrated V2527Engine Fan. In 54th AIAA Aerospace Sciences Meeting, 2016.

[11] H. Siller, A. Bassetti, W. Hage, and S. Funke. Measurements of the noise generated by a V2500 engine in flight and in static measurements on the ground. In 23rd AIAA/CEAS Aeroacoustics Conference. AIAA/CEAS, 2017.

[12] H. Siller, W. Hage, and T. Schumacher. Source localisation on aircraft in flight - new measurements with the DLR research aircraft Airbus 320 ATRA. In 7th Berlin Beamforming Conference, 2018.

[13] M. Pott-Pollenske, W. Dobrzynski, H. Buchholz, B. Gehlhar, and F. Walle. Validation of a semiempirical airframe noise prediction method through dedicated A319 flyover noise measurements. In 8th AIAA/CEAS Aeroacoustics Conference, 2002.

[14] ISO 9613-1. Acoustics - Attenuation of Sound during Propagation Outdoors (Part 1). Technical report, International Organization for Standardization (ISO), 1993.
[15] H. E. Bass, L. C. Sutherland, and A. J. Zuckerwar. Atmospheric absorption of sound: Update. The Journal of the Acoustical Society of America, 88(4):20192021, 1990.

[16] M. F. Heidmann and C. E. Feiler. Noise comparisons from full-scale fan tests at NASA Lewis research center. Technical report, NASA, 1973.

[17] K. Becker, K. Heitkamp, and E. Kügeler. Recent progress in a hybrid-grid CFD solver for turbomachinery flows. In 5th European Conference on Computational Fluid Dynamics, 2010.

[18] F. R. Menter. Two-equation eddy-viscosity turbulence models for engineering applications. AIAA Journal, 32(8):1598-1605, 1994.

[19] F.-S. Lien and M. A. Leschziner. Upstream monotonic interpolation for scalar transport with application to complex turbulent flows. International Journal for Numerical Methods in Fluids, 19(6):527-548, 1994.

[20] R. Schnell. Numerische Simulation des akustischen Nahfeldes einer Triebwerksgebläsestufe. Dissertation, TU Berlin, Berlin, 2004.

[21] C. Frey, G. Ashcroft, H.-P. Kersken, and C. Voigt. Simulations of Unsteady Blade Row Interactions using Linear and Non-linear Frequency Domain Methods. Journal of Engineering for Gas Turbines and Power-Transactions, 2015.

[22] A. Holewa, S. Lesnik, G. Ashcroft, and S. Guérin. CFD-Based Investigation of Turbine Tonal Noise Induced by Steady Hot Streaks. International Journal of Turbomachinery, Propulsion and Power, 2(1):3, 2017.

[23] S. Guérin, M. Vogt, and A. Holewa. Multiple Scattering of Acoustic Waves in Potential Mean Flow With Circumferential Distortion. In 23rd AIAA/CEAS Aeroacoustics Conference, 2017.

[24] C. Kissner, A. Holewa, and S. Guérin. Tonal Fan Noise Simulation of a V2527 Aircraft Engine: A Quantitative Assessment of a Best Practice Mesh. In $D A G A, 2017$.

[25] A. G. Wilson and J. Coupland. Numerical Prediction of Aeroengine Fan Stage Tone Noise Sources using CFD. In European Congress on Computational Methods in Applied Sciences and Engineering, 2004.

[26] J. de Laborderie, L. Soulat, and S. Moreau. Prediction of Noise Sources in Axial Compressor from URANS Simulation Journal of Propulsion and Power, 30(5):1257-1271, 2014.

[27] M. Daroukh, S. Moreau, N. Gourdain,J.-F. Boussuge, and C. Sensiau. Effect of Distortion on Turbofan Tonal Noise at Cutback with Hybrid Methods International Journal of Turbomachinery, Propulsion and Power, 2(3):16, 2017.

[28] J. M. Tyler and T. G. Sofrin. Axial flow compressor noise studies. Technical report, SAE Technical Paper, 1962.

[29] A. Wohlbrandt, C. Weckmüller, and S. Guérin. A robust extension to the triple plane pressure mode 
matching method by filtering convective perturbations. International Journal of Aeroacoustics, 15(12):41-58, 2016.

[30] C. L. Morfey. Sound transmission and generation in ducts with flow. Journal of Sound and Vibration, 14(1):37-55, 1971.

[31] M. Roger and H. Arbey. Relation de dispersion des ondes de pression dans un écoulement tournant. Acta Acustica united with Acustica, 59(2):95-101, 1985.

[32] V. Masson, H. Posson, M. Sanjosé, S. Moreau and M. Roger. Fan-OGV interaction broadband noise prediction in a rigid annular duct with swirling and sheared mean flow. In 22nd AIAA/CEAS Aeroacoustics Conference, 2016.

[33] S. Kaji and T. Okazaki. Propagation of sound waves through a blade row: II. Analysis based on the acceleration potential method. Journal of Sound and Vibration, 11(3):355-375, 1970.

[34] D. W. Bechert. Sound absorption caused by vorticity shedding, demonstrated with a jet flow. Journal of Sound and Vibration, 70(3):389-405, 1980.

[35] L. Heidelberg. Fan Noise Source Diagnostic Test Tone Modal Structure Results. In 8th AIAA/CEAS Aeroacoustics Conference, 2002.

[36] S. Guérin. Farfield Radiation of Induct-Cutoff Pressure Waves. In 23rd AIAA/CEAS Aeroacoustics Conference, 2017.

[37] A. Holewa, C. Weckmüller, and S. Guérin. Impact of bypass duct bifurcations on fan noise. Journal of Propulsion and Power, 30(1):143-152, 2013.

[38] S. Redonnet and Y. Druon. Computational Aeroacoustics of Aft Fan Noise Characterizing a Realistic Coaxial Engine. AIAA Journal, 50(5):1029-1046, 2012.

[39] J. Winkler, C. A. Reimann, C. D. Gumke, A. A. Ali, and R. A. Reba. Inlet and Aft Tonal Noise Predictions of a Full-Scale Turbofan Engine with Bifurcation and Inlet Distortion. In 23rd AIAA/CEAS Aeroacoustics Conference, 2017.

[40] X. Chen, X. Huang, and X. Zhang. Sound Radiation from a Bypass Duct with Bifurcations. AIAA Journal, 47(2):429-436, 2009.

[41] H. Namgoong and L. Arisetti. Fan OGV/ESS Interaction Tone Noise Analysis Using Linearized NavierStokes Equations. In 23rd AIAA/CEAS Aeroacoustics Conference, 2017.

[42] S. Guérin and A. Holewa. Fan tonal noise from aircraft aeroengines with short intake: a study at approach. to appear in the International Journal of Aeroacoustics, 2018.

[43] A. McAlpine and M. C. M. Wright. Acoustic scattering by a spliced turbofan inlet duct liner at supersonic fan speeds. Journal of Sound and Vibration, 292(3-5):911-934, 2006.

[44] A. Bassetti, H. Siller, and L. Enghardt. Numerical and experimental investigation of mode scattering at a fan casing liner. In 18th AIAA/CEAS Aeroacoustics Conference, 2012.
[45] Y. Özyörük, E. Alpman and V. Ahuja and L. N. Long. Frequency-domain prediction of turbofan noise radiation. Journal of Sound and Vibration, 270:933-950, 2004.

[46] C. Richter, J. A. Hay, L. Panek, N. Schönwald, S. Busse and F. Thiele. A review of time-domain impedance modelling and applications. Journal of Sound and Vibration, 330:3859-3873, 2011.

[47] E. Loukis, P. Wetta, K. Mathioudakis, A. Papathanasiou, and K. Papailiou. Combination of Different Unsteady Quantity Measurements for Gas Turbine Blade Fault Diagnosis. In ASME International Gas Turbine and Aeroengine Congress, 1991.

[48] W. Jürgens, U. Tapken, O. Lemke, I. Röhle, and L. Enghardt. Acoustic Localization of Vane Faults in Turbomachinery Based on Source Modeling. In 20th AIAA/CEAS Aeroacoustics Conference, 2014.

[49] M. Mößner, C. Kissner, J. Delfs, and L. Enghardt. Computational chain for virtual fly-over simulations applied to fan noise. In 24th AIAA/CEAS Aeroacoustics Conference, 2018. 